\title{
Qu'est-ce que l'art chorégraphique peut montrer du corps migrant ? Une étude des chorégraphies Ethnoscape de Cécile Proust et Rester. Étranger de Barbara Manzetti
}

\author{
Mélanie MESAGER, Université Paris 8
}

\begin{abstract}
Dans les années 1990, les corps de la danse ne sont pas réels pour moi : ils sont sophistiqués et formatés pour amener des formes, des idées et des pensées beaucoup plus abstraites. A cette époque, j'avais besoin de la réalité et les figurants me permettaient de questionner le corps de la danse. Mais maintenant, on peut être beaucoup plus expansif et dire «le corps de la scène ». Sur scène, les corps de ceux qui ne sont pas professionnels ne sont pas doués pour rendre les idées représentées, ils sont eux-mêmes les idées. Les idées sont le peuple, le boulanger, etc. (La Ribot et Cordeiro 27)
\end{abstract}

A l'horizon de la question de la migration sur la scène chorégraphique, il y a une volonté, qu'expriment certains chorégraphes actuels comme La Ribot, de décentrer la danse du corps du danseur, pour amener sur scène une « réalité » qui se trouverait dans les autres corps, ceux qui ne dansent pas, qui sont engagés dans le «monde». Il y a également, comme l'a remarqué le philosophe Frédéric Pouillaude, celle d'inscrire la danse dans un discours politique et social : «Ici et là apparaissent des tentatives d'ouvrir la scène chorégraphique à la présentation directe d'événements historiques et sociaux, généralement violents ou même tragiques, afin d'articuler le savoir kinesthésique et les procédés chorégraphiques dans notre existence politique contemporaine $»^{1}(80$, je traduis).

Comment le sujet de la migration questionne-t-il (et est-il questionné par) ce que La Ribot nomme le «corps de la scène »? A la fois inscrit dans une actualité particulièrement polémique quant à l'accueil qu'un pays se doit d'octroyer ou non à des personnes fuyant leur propre territoire, et dans des questionnements éthiques et humains, un tel sujet, lorsqu'il se fait chorégraphique, touche à la question de l'altérité corporelle. Or, quelles politiques sous-tendent l'acte de montrer le corps de l'autre ? Un tel acte artistique instaure-t-il nécessairement la dichotomie que postule la citation en exergue entre des corps «professionnels » qui se situeraient au niveau de la maîtrise de la représentation, et d'autres corps, qui seraient les corps que l'on montre, objets du discours et de l'acte artistique ?

L'article que je propose, pose ces questions par rapport à une œuvre particulière, Ethnoscape de Cécile Proust ou, plutôt, quant à deux œuvres, car j'y évoquerai également, comme un contrepoint, le projet Rester. Étranger de Barbara Manzetti. La confrontation de ces deux propositions chorégraphiques interroge les modalités de présence des corps migrants dans des œuvres qui en font leur sujet. 
Dans la note d'intention qui présente Ethnoscape sur le site femmeuses de Proust, on peut lire l'affirmation suivante : «Afin de partager avec des migrants l'espace de visibilité, de représentation et de parole qu'est le plateau, Cécile Proust et le vidéaste Jacques Hœpffner mettent en œuvre une série de rencontres et d'entretiens qu'ils filment ». S'il s'agit de «partager » un espace, comment les corps de ces «migrants » sont-ils rendus présents ? Comment s'effectue ce «partage» entre des présences différées, projetées sous forme de vidéos, et le corps de la chorégraphe qui parle et danse pendant la représentation ? Ces questions sont impossibles à dissocier des conditions de réception de la pièce, car la présence des corps que nous y voyons dépend de nos attentes (a fortiori dans le cas d'une chorégraphie se réclamant d'un sujet actuel aussi polémique que l'accueil des migrants); y proposer des éléments de réponse suppose de prendre en compte ma relation au travail de Proust.

La pièce que j'ai vue pour la première fois à la mairie du troisième arrondissement à Paris, était une chorégraphie d'une heure, dans un petit espace où les spectateurs étaient assis tout autour de l'espace de danse. Proust commençait par demander aux spectateurs de décliner leurs lieux de naissance, puis présentait différents documents vidéo qui étaient projetés aux murs. Entre ces projections se déployaient des moments de danse et de musique. Dans une série de représentations ultérieures, au Local, dans le douzième arrondissement de Paris, Proust a rajouté à sa chorégraphie une seconde partie durant laquelle les spectateurs étaient invités à discuter, avec des spécialistes invités, de la question de la migration, et j'étais alors chargée d'animer ces débats. Entre temps, j'ai discuté longuement avec Proust et mené des entretiens sur son travail.

Prise dans les discours sur la crise migratoire, la chorégraphie a eu diverses réceptions : si le public s'est parfois questionné sur la légitimité qu'avait Proust à convoquer le sujet de la migration, il a également pu être séduit par l'engagement politique de la pièce et la force de la présence scénique de la danseuse ${ }^{2}$. Partir de ces impressions contradictoires et réfléchir à ce qu'elles doivent aux modes de présence des corps dans la chorégraphie. sera une première façon d'interroger la relation de cette œuvre au migrant «étranger », à cet «autre corps » qu'elle montre sur la scène ${ }^{3}$. C'est alors qu'il sera intéressant, dans un second temps, de faire un détour par le projet Rester. Étranger de Manzetti à la Ménagerie de verre, qui proposait un mode de présence très différent ${ }^{4}$. Cependant, les discussions que j'ai eues avec la chorégraphe, la connaissance de la genèse d'Ethnoscape et la prise en compte de son évolution actuelle me permettent de poser sur cette œuvre un autre regard qui en déplace les enjeux, dès lors que les corps migrants ne sont plus seulement ceux des réfugiés politiques, mais également ceux des spectateurs et celui de la danseuse. Dans cette dernière perspective, j'essaierai de montrer que 
la forme de la chorégraphie nous parle sensiblement, au moins autant que son contenu référentiel, de migration. Mon propos sera donc de montrer comment une œuvre chorégraphique peut traiter du sujet de la migration, à la fois en s'inscrivant dans les discours polémiques de son époque, mais également, et peut-être plus encore, en se faisant lieu de rencontre entre les corps.

\section{Partager l'espace : les corps des migrants sont-ils présentés ou «absentés » dans Ethnoscape?}

Lors des représentations d'Ethnoscape à Paris, l'occupation scénique se répartissait entre trois modes de présence :

1) La diffusion de documents de plusieurs natures :

- Des documents vidéo, parmi lesquels certains étaient des récupérations de diffusions médiatiques, telles une intervention de Fatou Diome diffusée sur France 2 et une prise de parole de Madame le maire de Bassano del Grappa diffusée sur mediapart.fr. On voyait également l'extrait d'un documentaire sur la migration d'oiseaux sauvages. Et enfin des entretiens réalisés par Proust et Hoepffner, dans lesquels les personnes interrogées répondaient à la question «Qu'avez-vous perdu et gagné en quittant votre pays ? », puis étaient invitées à offrir un chant et une danse. Les entretiens étaient en français, ce qui signifie que les interlocuteurs étaient installés en France depuis suffisamment longtemps pour en connaître la langue, et on n'y entendait pas les questions posées.

- Des discours et lectures assumés par Proust, tels que la fausse lettre du maire de Calais rédigée par le collectif PEROU, un discours fictif dans lequel cette élue aurait demandé aux habitants de sa ville de considérer la jungle non pas comme un problème, mais comme une chance.

- Deux chansons diffusées dans leur version d'origine, l'une, malienne, de Rokia Traore, et l'autre, algérienne, de Khaled.

2) La présence de Proust, qui parlait, présentait les documents, s'adressait au public et assumait par là le rôle de narratrice. Elle parcourait également la salle dont elle occupait le centre, tamisait de l'argile sur le sol, qui recueillait ensuite la trace de ses passages. A trois reprises, Proust effectuait des mouvements dansés sur les chansons précédemment citées et la fausse lettre du maire de Calais.

3) Le public était également convié à prendre la parole. Au début de la représentation, chaque spectateur était invité à dire où il était né, et la liste des lieux était diffusée plus 
tard dans le spectacle. Dans les dernières représentations de décembre 2016, au Local, puis en novembre 2017 à l'école normale supérieure de la rue d'Ulm, un débat d'idées auquel les spectateurs pouvaient participer, faisait partie intégrante du spectacle. Proust a, par exemple, invité la géographe Anne-Laure Amilhat Szary, à parler de son ouvrage Qu'est-ce qu'une frontière aujourd'hui?

Les seules présences directes dans ce partage de l'espace sont donc celles de Proust et de son public. Les autres sont des présences différées, transmises au moyen de la vidéo, de la citation ou de la chanson. Parmi elles, il y a des personnes qui commentent les politiques européennes adoptées face à la migration et qui préconisent l'ouverture des frontières, et d'autres que l'on identifie comme des immigrées. Elles parlent le français, avec un léger accent, trace corporelle de leur étrangeté passée, au sein même de leur langage. Une personne comme Diome assume les deux statuts, elle se dit immigrée et parle de l'immigration actuelle. Les deux chansons introduisent un plurilinguisme dans le spectacle et convoquent des corps étrangers et des ailleurs par les inflexions de la voix. Au sein des discours eux-mêmes, sont évoqués les corps des migrants, notamment à travers la mention de séquelles, de morts, d'affects douloureux (la nostalgie, dans le discours d'un des témoins, le sentiment d'invisibilité dans un autre). Dans cette configuration, la chorégraphe et danseuse, qui se charge de la narration, apparaît comme la maîtresse d'un discours polyphonique dont elle maîtrise le sens en assumant le choix des documents présentés et leur mise en relation au cours du spectacle.

Une distance se creuse rapidement entre le corps de Proust et ceux qui sont évoqués par le langage, les chansons et les vidéos. Pour tenter de saisir à quoi tient ce contraste dans les modes de présence, portons notre regard sur deux séquences situées au début et à la fin de la représentation. Dans la première, la chorégraphe prend la parole et récite ce texte :

L'accord de Dublin, signé en 2003, stipule que les personnes voulant demander l'asile doivent le faire uniquement dans le premier pays européen où elles arrivent. Et pour ça les autorités ont le droit de prendre leurs empreintes digitales. A partir de ce moment-là, les migrantes et les migrants ont leur frontière au bout des doigts. Afin d'effacer cette frontière incorporée, des hommes et des femmes se brûlent la pulpe des doigts ${ }^{5}$.

A cet énoncé succède une danse durant laquelle Proust trace un ample cercle avec le bras droit, puis réunit ses deux mains au niveau du ventre, doigts contre doigts. Le mouvement est effectué deux fois, dans le silence, et le moment de contact entre le bout des doigts est suspendu, ralenti. Puis s'élève une musique, la voix de Traore qui chante Kolokani, et la phrase dansée se développe plusieurs fois en amples cercles du haut du corps, se terminant toujours par ce léger contact, cet auto-effleurement du bout des doigts. 
Dans le discours, les migrants sont évoqués par le pluriel : «les migrantes et les migrants », «des hommes et des femmes », et leur corps est marqué par l'automutilation, infligée pour contrer une loi qui les oblige à s'arrêter, qui empêche donc les corps de circuler, de migrer. Le lieu de cette automutilation, le bout des doigts, est repris sémantiquement par les mouvements dansés, sur le mode de l'euphémisme : à la brûlure auto-infligée répond un toucher ténu, léger. Regarder, sentir, vivre cette danse infléchit la façon dont nous construisons, en tant que spectateurs, la signifiance du texte qui vient d'être dit, et vice versa : la tragédie de la mutilation est confrontée à une danse d'une certaine douceur, faite d'accélérations et de suspensions temporelles, qui pourrait, dans un autre contexte, résonner différemment, mais qui porte ici une charge mélancolique.

Cette danse engage principalement le haut du corps, elle se déploie dans l'espace scénique par de légers déplacements marchés et tournés, parfois de petits sauts. La corporéité dansante est intègre, dans le sens où le corps reste toujours très structuré : les cambrés et les torsions se font sur l'axe vertical de jambes stables, les mouvements vers le sol sont retenus (le flux est condensé, évitant la chute, et les appuis sont bien fixes). Le rapport du corps à la gravité est parfaitement contrôlé, ainsi que celui à l'espace : dans les mouvements de rotation, la danseuse maîtrise son point de départ et d'arrivée, jamais elle ne se laisse entraîner par son propre mouvement. On ne ressent donc aucune dislocation dans la forme du corps ni de risque de déséquilibre. Dans cette première partie, la qualité de la danse contraste donc très fortement avec le corps évoqué dans le discours marqué par la blessure et la violence : le corps qu'on voit sur scène n'est en aucun cas un corps désarticulé ou blessé, dans sa gestuelle on ne ressent nulle violence ni saccade, mais de la fluidité, de l'intégrité.

A la fin du spectacle, c'est un autre contraste qui s'opère. Sur un écran vidéo apparaît un homme, Lounis, un des immigrés interrogés, qui effectue des mouvements de bras accompagnés de déplacements. La danse est touchante parce qu'elle semble incertaine, essentiellement en raison des transitions entre les mouvements qui ne sont pas fluides, de l'espace qui est un peu flou (les directions ne sont pas précises et semblent subies). L'équilibre, surtout dans les transitions de la station debout et à genoux, semble négocié avec la pesanteur, et l'ensemble du corps n'est pas engagé dans les gestes qui ont quelque chose de non abouti (le cou et la tête restent notamment statiques pour garder l'équilibre). On reconnaît dans ce corps un corps «piéton » (25), dans le sens où le définit Isabelle Ginot. Proust reprend de cette vidéo les mouvements de bras, et entame une magnifique danse orientale, particulièrement belle et maîtrisée, dont on peut admirer la coordination rythmique (la fluidité du rythme des bras en contrepoint des tremblements saccadés du bassin et du rythme de la marche sont 
particulièrement virtuoses, le tout parfaitement en rythme avec la chanson Wahrane de Khaled). Le contraste que je souligne ici n'est pas violent, il ne se joue pas dans l'affrontement. Dans la dynamique du spectacle, le corps dansant de Lounis est émouvant, et la danse de Proust est belle, elle nous transporte par son rythme et l'engagement de la danseuse dans le mouvement. Incontestablement, Proust séduit et s'allie ses spectateurs, et ce point, essentiel pour saisir les enjeux de la chorégraphie, sera traité plus loin.

Pour le moment, je pose la question suivante, issue de la confrontation des corps : qu'est-ce que cela signifie dire et montrer la violence faite à des corps humains en affichant soi-même une corporéité évoquant l'apaisement, la maîtrise - soit une certaine intégrité corporelle liée à la stabilité et au confort ? Pris en mauvaise part, cela peut poser un problème, celui de la légitimité qu'il y a à s'emparer d'un discours sur la migration : on peut ressentir un certain malaise à voir ce corps dansant présenter ces corps étrangers, soumis aux épreuves d'une migration forcée.

Cependant, les corps des immigrés ne sont pas seulement des corps en souffrance. Diome, dans l'émission dont un extrait est projeté, triomphe de son interlocuteur, Thierry Baudet : elle monopolise la parole dans une brillante harangue saluée par les rires bienveillants des invités, et Baudet est bien en peine de pouvoir répondre. Lors des représentations d'Ethnoscape auxquelles j'ai assisté, ce discours a toujours été salué des rires et des applaudissements du public de Proust. En réalité, le contraste est toujours le même : quand bien même Diome serait plus légitime que Proust à parler de migration, étant elle-même immigrée et se revendiquant comme telle, il y a en abîme ce même contraste entre un corps triomphant, celui du locuteur, visible sur scène ou sur un plateau de télévision, et le corps dont on parle, toujours à la troisième personne, ce corps marqué par la souffrance, qui n'est pas là, ou plus là, invisible, qu'on évoque seulement et qui nous renvoie à notre propre responsabilité quant à sa souffrance. Les médiations que sont la vidéo et le langage, ainsi que la diffusion de chansons, sont, certes, des moyens de «présenter » les corps absents ; mais en même temps, ce sont aussi des marques de leur absence, dans ce spectacle « vivant » ou d'autres corps sont bien présents. Et cette absence a un poids.

\section{Rester. Étranger de Manzetti : le choix de composer avec les corps présents}

Que serait-ce alors que de faire venir sur scène ces corps migrants, objets du discours ? D'autres propositions artistiques s'y sont essayées, et c'est notamment le cas de Rester. Étranger de Manzetti. A l'origine de ce projet chorégraphique, il n'y a, contrairement à Ethnoscape, pas de véritable volonté d'ouvrir une polémique sur le sujet de la migration, mais 
seulement un désir de rencontrer des personnes réfugiées en France, de proposer des actions qui leur sont utiles, et de faire de cette simple rencontre un acte artistique. Cependant, la chorégraphe s'est confrontée de façon violente aux risques que cette mise en présence pouvait présenter quant à la possibilité d'existence et de diffusion d'une telle œuvre.

Au rebours des formes spectaculaires de chorégraphie, Rester. Étranger accueillait, pendant le temps de résidence qui lui était accordé à la Ménagerie de verre, des personnes migrantes. Manzetti organisait pour celles-ci des cours de français, des ateliers d'écriture et leur proposait des temps de prises de parole. Les spectateurs étaient conviés à pénétrer dans une installation de longs rubans de papier déposés sur le sol et portant des inscriptions poétiques, et à assister, quand elles avaient lieu, aux diverses activités. Les immigrés avaient à ses yeux le statut d'artistes associés au projet et pouvaient se réunir selon leurs disponibilités, librement (de même que les spectateurs n'étaient jamais conviés à des horaires fixes, mais pouvaient aller et venir dans l'espace de la résidence artistique) ${ }^{6}$. Au terme de quelques mois, Manzetti s'est vu retirer la résidence, car la directrice a jugé que ces présences à la Ménagerie de verre gênaient le bon fonctionnement des lieux. C'est du moins ce qu'a dénoncé Gérard Mayen dans un article qui a été largement partagé dans le cercle des artistes chorégraphiques parisiens, choqués du racisme ordinaire dont cet acte a semblé soudain se faire l'étendard (13). Exerçant son droit de réponse, la directrice, Marie-Thérèse Allier, donne une version selon laquelle elle n'aurait demandé de libérer l'espace qu'à l'occasion d'une réunion avec les «institutions partenaires du lieu » (2). Cependant, son texte souligne également à deux reprises à quel point les corps migrants ont été introduits d'une façon ressentie comme clandestine, d'abord parce qu'elle précise que le projet de Manzetti «n'a pas pu acquérir le même statut que [les] deux artistes associés cette année. En effet, le dossier [...] monté ensemble pour obtenir des fonds de la Région Île-de-France dans le cadre d'une résidence d'auteur s'est vu recevoir une réponse négative. Cependant, Barbara a tout de même décidé de s’installer au rezde-chaussée de la Ménagerie » (1). Ensuite, la directrice se dit avoir été « enthousiaste à propos des ateliers d'écriture et des cours d'alphabétisation qu'elle [Manzetti] mettait en place auprès d'adolescents fraîchement arrivés sur le territoire français » (2), mais souligne ce changement : «[d]ans un premier temps, ces ateliers se déroulaient chez elle, jusqu'à ce qu'elle invite finalement les participants à la Ménagerie, quotidiennement, sans nous prévenir » (2). Dans ces phrases, qui pourtant sont écrites pour montrer la soi-disant tolérance de la directrice, on lit un sentiment de dépossession, de crainte dû au dépassement des limites institutionnelles : Manzetti s'est « tout de même » installée à la Ménagerie, bien qu'elle n'ait pas obtenu le statut de résidente officielle. Puis elle a en douce, « sans nous prévenir », investi la sphère publique 
de la Ménagerie au lieu de rester chez elle, comme si elle investissait un lieu qui lui était interdit, alors que tout porte à croire que son projet était toléré, bien qu'en marge du programme officiel, à la Ménagerie. Un tel reproche entre en contradiction avec la revendication d'enthousiasme précédente et ne s'explique que par la crainte de rendre visible quelque chose qui devrait rester caché, privé : les migrants qui auraient dû rester chez la chorégraphe. Il n'est pas anodin, dans ce sens, que la rupture finale se situe au moment de la réunion avec les «institutions partenaires »du lieu, soit à un moment de visibilité accrue. Ainsi, le danger ressenti qui a pu amener à l'exclusion, ne semblerait en aucun cas dû au propos de la création artistique ni aux intentions de l'auteur (Allier se dit « enthousiaste » au projet), mais bien à la présence des corps, et, plus encore qu'à leur présence effective, à la façon dont il est possible ou non d'en «contrôler» le flux et, sans doute, le sens : symboliquement, ces «migrants » étaient-ils perçus comme des artistes ? des sujets chorégraphiques? des «squatteurs » en situation irrégulière ? Ce flottement sémantique, ajouté à celui d'une présence potentiellement permanente, est en grande partie à la source de cet acte violent de destitution de la résidence qu'a accompli la directrice.

Revenant à Proust après ce détour, nous pouvons par contraste dire que, dans Ethnoscape, tout est sous contrôle : les corps étrangers sont filmés, leur flux de parole est maîtrisé, le sens est assumé par la narratrice. L’espace scénique, s’il est partagé, n'est pas occupé de façon égale, mais cette inégalité est au profit de la bonne diffusion de la chorégraphie, d'abord parce qu'elle en assure la légalité institutionnelle (elle n'introduit pas de corps en situation irrégulière, bien qu'elle dénonce, mais dans la sphère symbolique, les gouvernements qui ne les légitiment pas), ensuite parce que la temporalité de la diffusion d'un spectacle vivant, s'inscrivant dans des contraintes de lieu, de dates et de durée, n'est pas nécessairement compatible avec les priorités qui sont celles de réfugiés. Il aurait fallu adapter plus radicalement la forme même de la proposition chorégraphique, comme l'a fait Manzetti en proposant des cours de français comme acte artistique permanent dans un lieu donné7.

Nous pouvons donc à présent rapprocher la question de la légitimité de celle du mode de présence, et comprendre la gravité de ce qui s'y joue dans le milieu où la chorégraphie est présentée : le corps de Proust affirme un mode de présence intègre, maîtrisé, tout comme le discours qui se déroule durant le spectacle et dont la polyphonie est subordonnée à l'agencement narratif qu'elle assume. Le problème de la légitimité ne porte donc pas sur le fait que la chorégraphe se soit emparée (en tant que non-migrante) du sujet des migrations humaines; mais il peut plutôt être ressenti dans la forme de maîtrise des présences «migrantes » pendant la durée du spectacle : Proust a-t-elle ou non le droit (éthique) de 
s'emparer de ces discours sur les corps migrants sans assumer les risques qui s'attachent à leur présence effective? Sans doute, dans une certaine mesure, il est possible de répondre par la négative, ce qui explique le malaise décrit plus haut, le problème que pose la juxtaposition des corps.

Mais le temps différé et l'agencement des discours ne sont pas nécessairement ceux de l'appropriation et de la domination. On peut tout à fait y lire, en bonne part cette fois-ci, ceux du recul analytique, le choix d'une chorégraphie qui ne se lancerait pas dans la mêlée, mais amènerait plutôt à réfléchir sur le vécu de la migration et à porter un message politique en faveur de l'ouverture des frontières. Les documents réunis vont dans ce sens, et en particulier le choix de documents médiatiques passés : leur montage compose un point de vue, une thèse engagée assumée par la chorégraphe. Le dispositif ainsi envisagé ne vise pas à convaincre un public, car, comme le montrent les discussions durant la deuxième partie de la pièce, le public parisien qui assiste au Local à une représentation d'Ethnoscape, est très majoritairement, pour ne pas dire dans son intégralité, rallié aux causes défendues : l'ouverture des frontières, l'accueil des personnes migrantes et la libre circulation. La chorégraphie cherche plutôt à s'inscrire dans l'ordre de ces discours, à y prendre part, et à proposer une façon verbale et kinesthésique de penser ce point délicat de l'histoire contemporaine.

Ethnoscape est alors une chorégraphie qui présente du corps migrant une image magnifiée, où les mutilations et blessures ne sont pas celles de victimes, mais de héros exilés qui, comme le dit également Diome dans l'émission citée, bravent jusqu'à la mort pour franchir les frontières. Proust, dans une discussion que nous avons eue le 5 novembre 2016, dit au sujet de ses choix :

Dans Ethnoscape, j'essaie de ne pas travailler collée à cette violence et à cette souffrance trop extrême, mais à m'en décoller un peu, par choix optimiste, et parce que je ne veux pas montrer cette souffrance. Dans les pays qui reçoivent (ou qui ne reçoivent pas d'ailleurs), on aime les migrants comme des victimes, alors que ce sont des gens que je veux montrer comme des héros et comme des gens qui ont un projet, qui sont ambitieux et qui sont dans une capacité d'agir, une réalité vivante qui est celle qui m'intéresse. (Entretien inédit 5 novembre 2016)

Ethnoscape soutient donc la lutte des personnes engagées pour la cause de l'accueil des migrants et de l'ouverture des frontières. Le migrant reste l'Autre, venu d'ailleurs, mais un autre pris comme modèle, qui appuie un positionnement contre les politiques de fermeture, d'exclusion et de repli sur soi. La présence de la danseuse, particulièrement forte de ses jeux de regard et de la promiscuité avec les spectateurs, est alors fondamentale pour assurer la circulation du discours. Le plaisir que l'on prend à se voir, se sentir, s'entendre faire corps pour 
la cause commune de cette défense de l'autre n'est pas négligeable dans l'esthétique du spectacle. Il s'agit de celui de l'engagement politique, et également du désir d'être ensemble dont Charlotte Imbault a, par exemple, récemment souligné l'actualité sociale et artistique :

Le désir est au rassemblement, au sentiment océanique d'être ensemble. Alors qu'en France nous sommes en état d'urgence, il est urgent de se dire ensemble par tous les moyens. Et dans l'art : de pouvoir sentir l'autre, d'être au cœur et de travailler continuellement à la relation, cette fragilité invisible et pourtant si précieuse qui peut se mettre en place un à un, mais qui peut également, par un réseau d'opérations, travailler à la multiplication des liens. (104)

Malaise ou exaltation : ces deux affects naissent donc des mêmes éléments : le choix d'un mode de présence différé par le moyen du film (qui en mauvaise part usurpe la parole et en bonne part la magnifie, la cite, l'inclut dans une réflexion explicitement signée) ; la forte présence de Proust qui mène la danse avec maîtrise et s'inscrit, en tant qu'artiste chorégraphique, dans un ordre symbolique des discours sur la migration, inscription que partagent la plupart des spectateurs parisiens qui viennent assister à un tel spectacle. S'éloigner de ce contexte de réception, penser Ethnoscape autrement, peut nous amener à en déplacer les enjeux. Si nous quittons les sentiments contradictoires inhérents à ce contexte, entre méfiance ou gêne quant à la légitimité de la démarche et l'exaltation politique commune, cela nous permettra de chercher autre part le «corps migrant » et de réfléchir différemment à la façon dont il nous est rendu présent. Ce dernier temps s'appuie sur d'autres discours, dans lesquels se positionne également ma réception de la pièce : ceux de Proust, que j'ai moi-même provoqués, en l'interrogeant sur son travail. Il s'intéresse également à la chorégraphie à l'échelle de sa transformation dans le temps de l'intégralité de ses représentations.

\section{Comment un autre regard sur Ethnoscape permet d'en déplacer les enjeux}

Ethnoscape trouve son origine dans le projet Migrant Bodies, dont elle était, au départ, le journal de bord. Entre 2013 et 2015, un voyage à la rencontre de peuples migrants a été proposé à un groupe d'artistes chorégraphiques par plusieurs structures culturelles : la région Comune di Bassano del Grappa en Italie, La Briqueterie du Val-de-Marne en France, le Centre chorégraphique Circuit-Est au Québec, The Dance Centre en Colombie-Britannique et l'Institut croate de mouvement et de danse en Croatie. Lors d'une série de résidences dans les pays traversés, les chorégraphes présentaient des étapes d'un travail sur le thème du corps migrant.

Proust et Hoepffner étaient deux des artistes invités. Ils ont choisi, tout au long du voyage, de montrer leur travail, qui ne s'appelait alors qu'à peine Ethnoscape, dans des endroits 
périphériques aux lieux de spectacle: un vestiaire, une douche, le hall d'un théâtre, un ascenseur. Les documents présentés variaient selon les représentations ; ils étaient ceux qui documentaient le voyage et les réflexions qu'il a fait naître. Durant sa vie, la pièce Ethnoscape a duré de huit minutes à deux heures, elle s'est déployée dans des espaces très réduits ou beaucoup plus grands. Elle s'est laissé influencer chaque fois par l'endroit dans lequel elle était dansée : les mouvements se sont adaptés à la morphologie du lieu, la temporalité s'est modulée selon les circonstances.

Bien que cela ne soit pas visible lors d'une représentation donnée, la gestuelle de Proust porte les traces de ce voyage et de sa propre histoire quand elle s'expose dans l'espace d'un théâtre parisien. La première danse, que j'ai décrite plus haut, dans laquelle les doigts de la danseuse se rejoignent après un déploiement des bras, a été par exemple composée dans une salle de douche à Montréal, qui, selon les souvenirs de Proust, était juste assez large pour contenir son envergure les bras écartés. Une telle contrainte lui a inspiré cette danse du haut des bras et du buste, adaptée d'une technique de danse traditionnelle japonaise.

Cette imprégnation de l'ailleurs n'est pas nouvelle dans la démarche de Proust, qui, plus loin encore que le voyage Migrant Bodies, est allée, lors de nombreux séjours au Japon, en Afrique ou en Espagne, étudier des danses d'origines aussi diverses que le Jiuta Maï, le Kabuki, le Flamenco et les danses orientales. En 1995, lors d'un entretien accordé à Isabelle Launay, elle inscrivait cette recherche de l'ailleurs dans les perspectives féministes et la revendication d'un corps féminin sexué qui étaient les siennes (1-2). Ces mouvements qu'elle a traversés durant toute sa vie et incorporés, sont ceux qui composent la danse d'Ethnoscape. Au fil des années, ils sont devenus «sa » danse, formant à leur tour une matière documentaire, une strate corporelle de la circulation des rythmes, des mouvements, des énergies, des techniques du corps, qu'elle revendique :

C'est une envie de danser ailleurs, de danser comme on danse ailleurs, qui a structuré tout mon désir de danse. [...] Quand je visualise ma matière chorégraphique, je me vois dans des lieux qui sont pas mes lieux de naissance [...]. Comme dans Ethnoscape, puisque c'est ça aussi le thème global : ce monde qui est mouvant, qui est constitué de toutes ces migrations de divers niveaux. Les migrants qui n'ont pas le choix que de partir de chez eux, mais aussi la migration beaucoup plus légère, les migrations artistiques... enfin les «mouvements », ce serait beaucoup plus juste de parler de mouvements. Arjun Appadurai pense le monde en mouvement, et la façon dont ces mouvements, évidemment, modifient le monde, mais pas seulement là où ça bouge : ça modifie le monde pour ceux qui restent aussi. (Entretien inédit 29 septembre 2016) 
Dans ces propos, Proust met explicitement en parallèle la composition d'Ethnoscape et cette envie primordiale d'ailleurs, qui, on le voit maintenant, dépasse largement le contexte de la scène parisienne en 2016. Sous cet angle, la notion de «corps migrant» se déplace considérablement et s'élargit. Il s'agit à présent d'une corporéité migrante qui traverse non pas seulement la représentation des corps socialement marqués, et sémiotisés par nos regards comme étant des réfugiés politiques, mais tous les corps, celui de Proust, celui des spectateurs qui déclinent au début du spectacle leurs lieux de naissance. Et même, pourrait-on dire, plus que les corps eux-mêmes, la mise en relation entre ces corps, à savoir la chorégraphie, qui porte les marques de ces multiples voyages. La caractéristique de cette corporéité migrante serait alors une certaine dynamique, un élan vital de circulation et le développement d'une capacité d'adaptation à des environnements nouveaux. C'est d'ailleurs bien ce qu'évoque le titre de la chorégraphie, comme le rappelle Proust dans l'entretien cité en évoquant les idées de l'anthropologue Appadurai. Selon ce chercheur, le terme «ethnoscape » regroupe tous les mouvements de personnes à travers le monde, qu'ils soient des touristes, immigrants, réfugiés... flux qui redessinent constamment le paysage linguistique, politique, culturel du monde : l'environnement qui est pénétré n'a d'autre choix que de se laisser modifier par le milieu des personnes qui le traversent (69). De ce regard émerge une proposition chorégraphique revendiquant la liberté des flux circulatoires, insistant sur la vitalité du voyage et de l'échange.

La chorégraphie Ethnoscape, considérée plus largement, est donc une entité polymorphe dont une des caractéristiques serait de s'adapter, d'être en dialogue ou en porosité avec les espaces géographiques et humains qu'elle traverse, et d'en transporter les marques, au détriment d'une identité formelle et visuelle qui la définirait. La présence différée, dans cette perspective, acquiert une nouvelle valeur : celle de l'archive, de la trace portée par les vidéos ou par les corps d'un vécu antérieur.

Actuellement, il n'est pas anodin de souligner que la forme qui nous semble figée quand on en considère une représentation donnée, est encore en mutation, d'abord parce qu'au Local, en décembre 2016, Proust a ajouté la seconde partie entièrement composée d'une discussion entre les spectateurs et des expertes invitées à parler de la migration; ensuite parce que l'ordre des documents diffusés et la durée de la pièce sont encore très variables en fonction des lieux de représentation ; enfin parce que Proust et Hoepffner ont mené récemment, avec des étudiants de l'École nationale supérieure (ENS) de Paris et de l'Université Paris 8, un projet consistant à inventer des partitions d'Ethnoscape inspirées des scores d'Anna Halprin ${ }^{8}$ qui permettent à chacun des participants de s'approprier la démarche et de créer leur propre Ethnoscape. Ces 
partitions sont des protocoles de rencontre : comment aller «vers » des personnes qui vivent ou ont vécu des migrations, à Paris, ou à l'étranger. Il s'agit donc de partitionner un déplacement corporel et identitaire, et un échange de type conversationnel, en vue de créer une forme non définie, qui s'adapte à chaque rencontre.

Cet aperçu des mutations d'Ethnoscape nous montre que les formes présentées au public ne sont qu'une partie de l'œuvre. Si les corps «migrants » apparaissent de façon différée, médiés par la vidéo ou l'enregistrement, il n'en reste pas moins qu'une des préoccupations majeures du processus reste la rencontre avec l'autre, et la façon dont on peut la penser. La dichotomie entre présence directe et présence différée qui distingue le spectacle Ethnoscape de formes comme Rester. Étranger de Manzetti, se déplace : elle ne concerne plus tant le mode de présence des corps migrants que la temporalité de l'œuvre elle-même et son espace de représentation. Là où Manzetti amène dans un lieu de danse ces corps extérieurs, Proust sort du studio de danse pour aller trouver ces corps, croiser leur milieu, et en ramène des traces qu'elle montre sur scène. Il s'agit de deux façons de franchir les frontières. Le temps de la confrontation au public, chez Proust, est celui du récit au passé, qui peut de nouveau se rejouer dans le présent à travers une discussion, ou dont la composition peut se réinventer, par l'intermédiaire des scores, et se transmettre à d'autres. Il y a donc par-delà chaque représentation d'Ethnoscape cet horizon qui est celui d'une œuvre migrante et polymorphe.

Le terme de ce parcours ne propose pas de solution ni de prise de parti quant à l'efficience d'Ethnoscape à documenter la migration, et s'assume comme tel. Je pense en effet que cette solution n'existe pas : voir dans cette chorégraphie un processus d'appropriation de corps et de discours étrangers ou une œuvre politique efficace et exaltante appartient à chacun. L'exemple de Manzetti montre à quel point cette inscription contextuelle fabrique la portée de l'œuvre : alors qu'il s'agissait pour elle moins de permettre un « espace de visibilité » que de faire œuvre d'un vécu immédiat, son acte artistique acquiert une visibilité et prend place dans les discours actuels sur la mise à l'écart des étrangers au moment où Mayen publie l'article qui dénonce l'acte d'exclusion de la directrice et le qualifie de raciste. Proust, au contraire, postule un sujet polémique, mais qui se trouve apaisé et, pourrait-on dire, sécurisé par la forme choisie et le public qu'elle attire. Il y a comme un paradoxe qui se joue entre l'intention affichée des œuvres et leur portée contextuelle : l'œuvre de Proust se veut polémique et fait finalement consensus, alors que celle de Manzetti se retrouve, comme malgré elle, prise dans une violente polémique sur sa légitimité à être accueillie à la Ménagerie de verre, ce qui, par répercussion, interroge la capacité des institutions à accueillir des migrants dans des lieux de visibilité. Nous pouvons à présent dire que ce paradoxe tient au mode de présence des corps : rendre visible, 
intégrer l'autre dans nos systèmes de représentation, lui octroyer une place dans nos discours, comporte nécessairement ce revers, celui justement de le réduire au rang de discours, de représentation, et donc de le rendre absent.

Le changement d'angle de vue proposé, plus modestement qu'une «solution » aux problèmes insolubles que posent la présence et l'absence des corps des migrants évoqués dans la chorégraphie, me permet de proposer deux niveaux de lecture de ce corps attendu et convoqué. L'un, porté par la matière documentaire mise en scène par la chorégraphe, s'inscrit dans les discours politiques actuels sur la migration et dans la confrontation avec d'autres œuvres contemporaines ; on y perçoit des corps, magnifiés ou mutilés, qui nous renvoient à notre responsabilité par rapport aux corps étrangers et à l'accueil que nous leur réservons sur le territoire français. Ces corps entrent en opposition avec celui de Proust, qui, comme elle mène la danse, présente un corps intègre et maîtrise le discours, peut sembler manquer de légitimité à s'emparer de ces « autres voix », mais également, à la fois, prendre parti et partager des idées assumées et engageantes sur la migration et ses implications politiques. Le deuxième niveau est celui qui nous amène à penser l'œuvre Ethnoscape de façon plus étendue, dans la temporalité de son existence. Considérant que chaque représentation n'est finalement qu'une trace possible d'un voyage toujours à poursuivre, nous ne sommes plus tant en face d'une représentation du corps migrant que d'un croisement dynamique de corps modelant le monde par leurs rencontres. Le travail que Proust a mené à l'ENS et à l'Université Paris 8 est primordial en ce sens : cherchant à partitionner des circulations et des modalités de conversations et de rencontre, elle tend à positionner l'œuvre Ethnoscape au-delà des formes contingentes qu'elle a prises dans des espaces de représentation. Ce dont il s'agit, c'est de faire œuvre d'un mouvement de circulation des corps dans l'espace géographique, et c'est peut-être à cet endroit précis que la danse a quelque chose à partager avec la migration humaine et ses implications politiques. «Les idées sont le peuple, le boulanger, etc. » affirmait La Ribot dans l'entretien accordé à Cordeiro cité en exergue de ce texte. Les «idées » étaient alors portées par les corps et leurs fonctions socioprofessionnelles, alors que dans la perspective qui est celle de la fin de cet article, le sens de la chorégraphie se trouve moins dans la représentation sociale du corps que dans la rencontre et le mouvement qui relient entre eux les corps, ceux qui ont été rencontrés comme ceux qui se rencontrent à l'occasion d'une représentation : la chorégraphe, les spectateurs dans la multiplicité de leurs origines, les étudiants à qui l'on transmet un protocole et qui, à leur tour, font des rencontres et en présentent les traces. 


\section{Bibliographie}

Allier, Marie-Thérèse. « Droit de réponse ». Mouvement. 22 avril 2017. http://www.mouvement.net/fil-de-une/droit-de-reponse. Consulté le 12 décembre 2017.

Amilhat Szary, Anne-Laure. Qu'est-ce qu'une frontière aujourd'hui ? Paris : PUF, 2015.

Appadurai, Arjun. Après le colonialisme. Les conséquences culturelles de la globalisation. Tr. de 1'anglais Françoise Bouillot et Hélène Frappat. Paris : Payot, 2001, [1996].

Ginot, Isabelle. «Du piéton ordinaire ». Corps (in)croyables. Pratiques amateur en danse contemporaine. Dir. Michel Briand. Pantin : Centre national de la danse, 2017 : 25-43.

Halprin, Lawrence. «Les cycles RSVP. Dispositifs de création dans le champ des activités humaines ». De l'une à l'autre. Composer, apprendre et partager en mouvements. Bruxelles : Contredanse, 2010, [1970] : 8-33.

Imbault, Charlotte. «Do You Care?»Watt, danse et performance. Bayeux : White Spirit (2017) : 102-109.

La Ribot et Volmir Cordeiro. «La parole fait encore une autre chose, elle fait une mise en scène ». Watt, danse et performance. Bayeux : White Spirit (2017) : 6-37.

Launay, Isabelle. «Entretien avec Cécile Proust ». Mouvement 9. Février-mars 1995. www.danse.univ-paris8.fr. Consulté le 12 décembre 2017.

Mayen, Gérard. « Des migrants peu désirés ». Mouvement. 13 avril 2017. http://www.mouvement.net/analyses/enquetes/des-migrants-si-peu-desires. Consulté le 12 décembre 2017.

Pouillaude, Frédéric. «Dance as Documentary : Conflictual Images in the Choreographic Mirror (On Archive by Arkadi Zaides) ». Dance Research Journal 48.2 (2016) : 80-94.

\section{Entretiens inédits}

Proust, Cécile. Entretien inédit avec Mélanie Mesager. 29 septembre 2016.

---. Entretien inédit avec Mélanie Mesager. 5 novembre 2016.

\section{Chorégraphies citées}

Manzetti, Barbara. Rester. Étranger. 2017-.

Proust, Cécile. Ethnoscape. 2015-. 


\section{Liens Internet}

Entretien de Barbara Manzetti sur r22 au sujet de Rester. Étranger. https://r22.fr/resteretranger/. Consulté le 12 décembre 2017.

Présentation d'Ethnoscape sur le site femmeuses de Cécile Proust et Jacques Hœpffner. http://femmeuses.org/modxFem/index.php?id=41. Consulté le 12 décembre 2017

\footnotetext{
Notes

${ }^{1}$ Voici le texte original : «Here and there appear some attempts to open the choreographic stage to a direct presentation of historical and social events, generally violent or even tragic, in order to articulate the kinesthetic knowledge and the choreographic procedures in our contemporary political existence » (80).

${ }^{2}$ En France comme dans plusieurs pays d'Europe, la « crise migratoire » désigne l'arrivée massive de migrants fuyant leurs pays d'origine pour des raisons politiques. De nombreux discours concernant leur accueil ont cours, ainsi que sur la pertinence d'ouvrir les frontières à ces nouveaux arrivants. Entre peur de l'inconnu et de l'invasion et politiques de l'hospitalité, les affrontements sont nombreux et exacerbés. Mentionnant les réactions du public face au spectacle de Proust, je fais référence aux discussions informelles que j'ai pu avoir avec des spectateurs. La question de la « légitimité » dépasse largement cette œuvre et se pose dans le milieu du spectacle : peut-on en tant que Française et blanche s'emparer d'un sujet concernant des personnes migrantes ou appartenant à une minorité opprimée ? Un collectif d'artistes chorégraphiques, d'universitaires et de directeurs de lieux de spectacle, appelé « la permanence », s'est monté à Paris depuis 2017 pour réfléchir à ces questions.

3 J'entends par « mode de présence des corps » la façon dont les corps parlants, dansants, et les corps dont on parle sont évoqués sur la scène. Je distinguerai notamment dans la suite de l'article un mode de présence immédiat, et un mode de présence différé, qui transite par la captation vidéo, l'évocation linguistique ou l'enregistrement audio.

${ }^{4}$ Fondée en 1984 par Marie-Thérèse Allier, la Ménagerie de verre est un espace parisien dédié à l'art contemporain et plus particulièrement à la danse. Il accueille des artistes en résidence et propose des cours destinés aux danseurs. Rester. Etranger de Manzetti est un projet chorégraphique et littéraire en association avec des réfugiés politiques. Il s'agit pour la chorégraphe de faire œuvre de sa rencontre et des relations familiales qu'elle développe avec ces migrants.

${ }^{5}$ Il s'agit d'un texte écrit par Proust pour son spectacle. Les références des lois auxquelles elle fait allusion ne nous sont pas données.

${ }^{6}$ Manzetti parle de cette action artistique à Olivier Marboeuf le 2 février 2017 sur https://r22.fr/rester-etranger/. Elle revendique notamment le statut d'auteur pour tous les participants.

${ }^{7}$ Lors d'une des représentations au Local, Proust, en deuxième partie, a invité la comédienne Valérie Thomas, qui a déclaré abandonner la scène pour se consacrer à l'aide des migrants, ne pouvant, quant à elle, plus exercer d'activité symbolique dans ces conditions d'urgence. Thomas avait pour projet d'intervenir en association avec une personne réfugiée, qui n'a pas pu être présente, étant appelée à prendre le logement qu'elle sollicitait depuis des mois le soir même, sans retard. Cet événement a été pour nous l'occasion de discuter de ces questions de temporalité. Manzetti, dans l'entretien cité, précise qu'elle assure une permanence, invitant les migrants associés à venir quand ils veulent, sans obligation. Cette adaptation refonde la temporalité du spectacle vivant « traditionnel », qui implique un rendez-vous, en tant qu'événement ponctuel, très largement planifié entre des artistes, des techniciens, des spectateurs.

${ }^{8}$ Anna Halprin a adapté pour la chorégraphie les cycles RSVP de Lawrence Halprin, qui consistaient à structurer toute activité humaine selon quatre phases : «Ressources» (identification des matériaux), «Structures» (composition d'une partition), «Valuaction» (analyse des résultats et prise de décisions), «Performance » (réalisation de la partition) (8). Elle en a fait des scores (partitions) qui servaient de moteur à la création chorégraphique.
} 